\title{
3D UWB Tomography for Medical Imaging Applications
}

\author{
M. Guardiola ${ }^{*(1)}$, L. Jofre ${ }^{(1)}$, and J. Romeu ${ }^{(1)}$ \\ (1) AntennaLab, Universitat Politècnica de Catalunya, Barcelona, Spain \\ E-mail: marta.guardiola@tsc.upc.edu
}

\section{Introduction}

Biomedical applications have been an active research field for the development of microwave imaging due to the non-ionizing character of this radiation and its comfortable application. In particular, stroke detection, constitute a challenging application due to the high contrast between the mediums involved [1]. Ischemic strokes occur due to a loss of blood supply to a part of the brain caused by a blood clot, possibly leading to death of the tissue. Nowadays, stroke affects 795,000 people in US every year, meaning that, on average, every 40 seconds someone in the US has a stroke [2]. Moreover, it accounted for about one of every 17 deaths, representing 150,000 deceases per year in US. Currently, they are usually treated with thrombolytics (clot buster). However to ensure its effectiveness, it has to be given within 3 hours of the onset of symptoms and requires a firm diagnosis of an ischemic stroke. Currently, this diagnosis relies on imaging methods including CT, PET and MRI, which cannot be widely available at all medical centers due to its high cost. Hence, many times the diagnosis is delayed or not very accurate. Microwaves experiment highly sensitive interactions with dielectric properties of tissues, which can be used to identify them. This turns to be useful in stroke detection to distinguish between ischemic and hemorrhagic strokes, which present similar symptoms, but radically different treatment. Thus, by using microwave imaging, we will have enough specificity to distinguish vessels of blood in the brain (hemorrhagic stroke) and in this case, identify the injury as an ischemic stroke in a cost-effective way.

Nowadays most microwave imaging algorithms are based on 2D reconstructions. These reconstructions are not always reliable because they are unable to discriminate vertically. For this reason there is an increasing interest in $3 \mathrm{D}$ reconstruction algorithms for microwave imaging, mainly boosted by the advent of both efficient numerical approaches and more powerful PCs. Up to now, most studies have been limited to the use of iterative schemes [3], which have a high computational cost. This opens the door to less computationally intensive algorithms such as ones based on linearizing approximations [4] or UWB Hybrid Focusing techniques [5]. In this paper a novel 3D UWB tomographic algorithm based in [4] applied to stroke detection is presented. For its validation a realistic 3D brain numerical model is simulated and reconstructed to detect the existence of a hemorrhage.

\section{D UWB Hybrid Focusing Image Reconstruction Algorithm}

The algorithm presented here consists in the extension of previous 2D UWB techniques to 3D cylindrical geometry, see Fig. 1. As explained in [4], the tomographic algorithm relies on obtaining the dielectric contrast $C(\vec{r})$ of the scene, which is related to the complex permittivity of the object under test through:

$$
C(\vec{r})=1-\frac{\varepsilon(\vec{r})}{\varepsilon_{0}^{e x t}}
$$


where $\varepsilon_{0}^{\text {ext }}$ is the permittivity of the external medium. The spectrum of the contrast profile can be related to the measured scattered field at all probes when it is produced by an incident plane wave that illuminates the body under test [4].

By using a cylindrical geometry of measurement, plane waves have to be expressed as a combination of cylindrical waves emanating from the probes situated at $\left(\theta^{\prime}, z^{\prime}\right)$. In this case, the current distribution to produce a vertical plane wave towards $\vec{k}=k_{0}^{e x t}(\cos \theta \sin \phi \hat{x}+\sin \theta \sin \phi \hat{y}+\cos \theta \hat{z})$ is [6]:

$$
I\left(\phi^{\prime}, z^{\prime} ; \vec{k}\right)=-\frac{1}{2 \pi^{2}} e^{j k_{z} z^{\prime}} \sum_{n=1}^{k_{0} R} j^{n+1} e^{j n\left(\phi-\phi^{\prime}\right)} \frac{1}{\sin \theta H_{n}^{(2)}\left(k_{R} R\right)}
$$

Where $k_{0}^{\text {ext }}$ is the wavenumber in the external medium, $\mathrm{R}$ is the radius of the cylinder, $(\theta, \phi)$ is the direction of the plane wave, $k_{z}=k_{0}^{\text {ext }} \cos \theta$ and $k_{R}=\sqrt{\left(k_{0}^{\text {ext }}\right)^{2}-k_{z}^{2}}$. Fig. 3 shows the amplitude and phase of the synthesized plane wave electric field on the 3 main planes when the incidence is $\theta=90^{\circ}$ and $\phi=45^{\circ}$. The closer $\theta$ is to $0^{\circ}$ or $180^{\circ}$, the less accurate the plane wave is. This is due to the truncation error provided by the limited extent of the measurement surface in the $\mathrm{z}$ direction [6]. To avoid including unacceptable plane waves in reconstructions, $\theta$ has been limited between $45^{\circ}$ and $135^{\circ}$, resulting in a small degradation of the resolution in $\mathrm{z}$ direction.

The mathematical formulation of the algorithm is based on the reciprocity theorem (3). The spectrum of the induced currents in the object $\tilde{J}\left(\vec{k}_{2}\right)$ can be expressed by weighting the scattered field $\left(\vec{E}^{s}\right)$ measured on the array by the current distribution that would produce a plane wave in the direction of the vector $\vec{k}_{2}$ in the region occupied by the body under test $\left(\vec{J}^{a}\right),(2)$.

$$
\int_{v_{b}} \vec{J}(\vec{r}) e^{-j \vec{k}_{2} \vec{r}} d v_{b}=\int_{v_{a}} \vec{J}^{a} \vec{E}^{s} d v_{a}=\int_{v^{\prime} a} I\left(\theta^{\prime}, z^{\prime} ; \vec{k}_{2}\right) \vec{E}^{s} d v^{\prime}{ }_{a}
$$

Under Born approximation (the scattered field is negligible in front of the incident field $\vec{E}^{0}$ ), the induced current, can be expressed in terms of the contrast profile as:

$$
\vec{J}(\vec{r}) \cong j \omega \varepsilon_{0}^{\text {ext }} C(\vec{r}) \vec{E}^{0}\left(\vec{r} ; \vec{k}_{1}\right) \stackrel{\mathcal{F}}{\Rightarrow} \vec{J}(\vec{\eta}) \cong j \omega \varepsilon_{0}^{\text {ext }} \tilde{C}\left(\vec{\eta}-\vec{k}_{1}\right)
$$

Where $\vec{E}^{0}=e^{-j \vec{k}_{1} \vec{r}}$ can be expressed again by weighting transmitted signals with $I\left(\theta^{\prime}, z^{\prime} ; \vec{k}_{1}\right)$.

Finally, considering $\vec{E}^{s}$ the scattered field produced by $\vec{E}^{0}$ and combining (2), (3) and (4), the spectrum of the contrast can be expressed as:

$\tilde{C}\left(\vec{k}_{1}+\vec{k}_{2}\right)=\frac{1}{j \omega \varepsilon_{0}^{\text {ext }}} \sum_{i=1}^{N_{T}} \sum_{j=1}^{N_{R}} \vec{E}^{s}\left(\phi_{T_{i}}^{\prime}, z_{T_{i}}^{\prime} ; \phi_{R_{j}}^{\prime}, z_{R_{j}}^{\prime}\right) \cdot I\left(\phi_{T_{i}}^{\prime}, z_{T_{i}}^{\prime} ; \vec{k}_{1}\right) I\left(\phi_{R_{j}}^{\prime}, z_{R_{j}}^{\prime} ; \vec{k}_{2}\right)$

The spectrum of the contrast is obtained on shifted spheres of radius $k_{0}^{\text {ext }}$. This shift depends on the direction of the plane waves and thus, by using different directions of incidence, the spectrum can be finely sampled inside of a sphere of radius $2 k_{0}^{\text {ext }}$. Additionally, by using a number of frequencies of illumination, the radius of the spheres changes, enhancing the reconstruction. Multi-frequency combination has been implemented in this algorithm as the summation of the magnitude of mono-frequency 
images. This solution is widely applicable and preserves the shape and size of reconstructed objects in cases where coherent combination does not work [5].
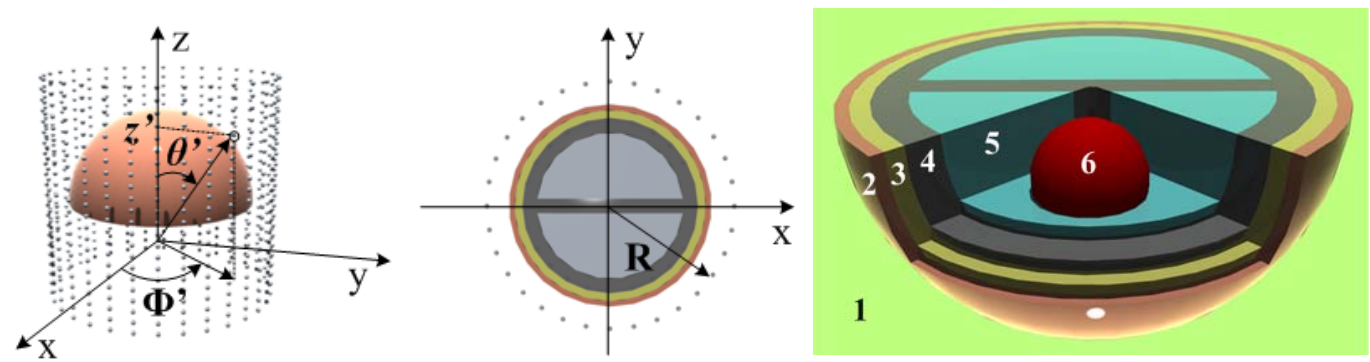

Fig. 1: 3D view and $\mathrm{XY}$ plane of the measurement geometry. In black the coordinate system for the plane waves and the antennas position are represented.
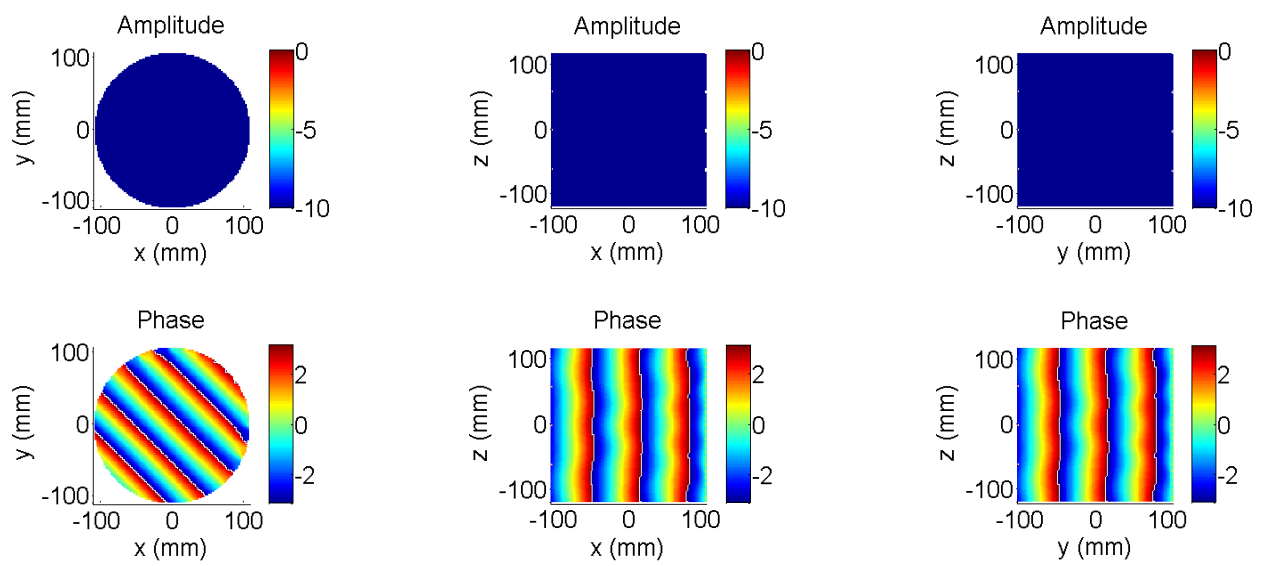

Plane XY

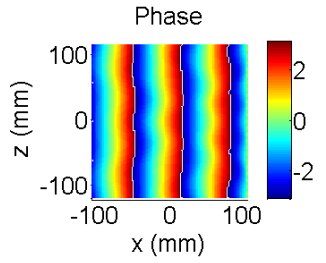

Plane XZ
Fig. 2: Simulated 3D model of the head.

Fig. 3: Three main planes of the synthesized plane wave in the direction $\theta=90^{\circ}$ and $\phi=45^{\circ}$

\section{Preliminary Results of Brain Stroke Detection}

The assessment of the algorithm is accomplished by using computer simulations of a simplified 3D human head model. The model includes several layers corresponding to the skin, the skull and the brain itself, composed by the grey and white matter. The hemorrhagic stroke injury is simulated as a sphere of blood inserted in the white matter, see Fig. 2. The dielectric properties of the model are summarized in Table 1. Additionally, an external matching medium of $\varepsilon_{\mathrm{r}}=40$ has been placed in order to reduce the initial contrast. When the external medium presents such large permittivity, it is possible to use low frequencies while maintaining a good resolution. In this case, the imaging algorithm is assessed using a frequency range between $0.3-1 \mathrm{GHz}$, which corresponds to a resolution of $2 \mathrm{~cm}$. The scanning system consists of both transmitters and receivers placed along the surface of a cylinder of $22 \mathrm{~cm}$ in diameter, composed by 25 rings of 32 antennas separated $1 \mathrm{~cm}$ in $\mathrm{z}$ direction. Fig. 4 shows the results of the reconstruction. It can be seen that in spite of the significant contrast between layers, the algorithm is able to retrieve the position and shape of the whole brain and specially of the blood vessel. 


\begin{tabular}{lcccccc}
\hline & $\begin{array}{c}\text { Matching } \\
\text { medium }\end{array}$ & Skin & Skull & $\begin{array}{c}\text { Grey } \\
\text { matter }\end{array}$ & $\begin{array}{c}\text { White } \\
\text { matter }\end{array}$ & Stroke \\
\hline Number & 1 & 2 & 3 & 4 & 5 & 6 \\
\hline External radius & & $90 \mathrm{~mm}$ & $85 \mathrm{~mm}$ & $87 \mathrm{~mm}$ & $65 \mathrm{~mm}$ & $2 \mathrm{~mm}$ \\
\hline Permittivity & 40 & 40 & 13 & 50 & 40 & 50 \\
\hline
\end{tabular}

Table 1: Properties of the model of the head [1], [7] at $1 \mathrm{GHz}$. At the current stage of development losses have been neglected.
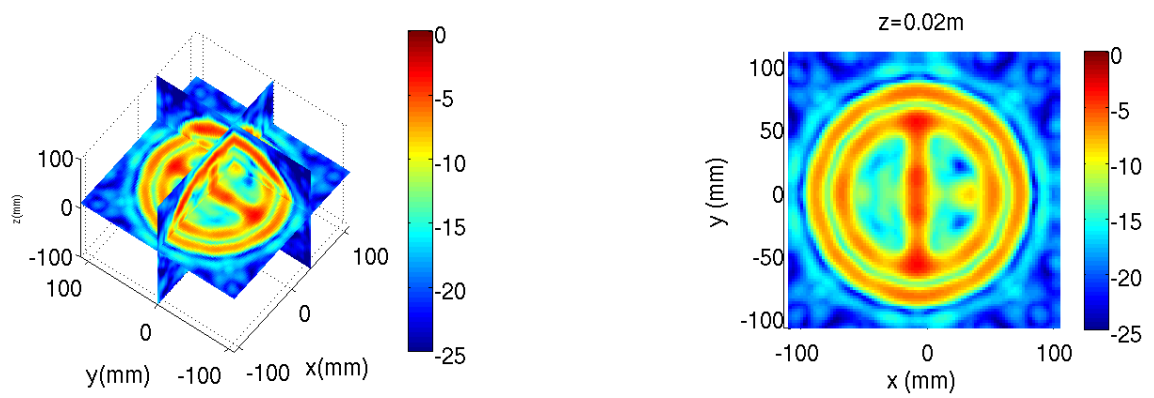

Fig. 4: 3D view and horizontal cut corresponding to the position of the blood vessel.

\section{Conclusions}

In this paper, a novel 3D UWB tomographic algorithm has been presented for brain hemorrhagic stroke detection. While 2D reconstructions are unable to distinguish between features at different heights, 3D reconstructions allow to obtain a complete representation of the brain and thus to differentiate them. A human brain model has been successfully reconstructed and the position of a blood vessel detected.

\section{Acknowledgments}

This work was supported in part by the Spanish Interministerial Commission on Science and Technology (CICYT) under projects TEC2007-66698-C04-01/TCM and CONSOLIDER CSD2008-00068.

\section{References}

[1] S. Y. Semenov, and D. R. Corfield, "Microwave Tomography for Brain Imaging: Feasibility Assessment for Stroke Detection," International Journal of Antennas and Propagation, vol.2008, no., pp., 2008.

[2] (2009) Heart disease and stroke statistics - 2009 Upgrade at-a-glance. [Online]. Available: http://www.americanheart.org

[3] J. De Zaeytijd, A. Franchois, C. Eyraud, and J. Greffin, "Full-Wave Three-Dimensional Microwave Imaging With a Regularized Gauss-Newton Method - Theory and Experiment," IEEE Trans. Ant. Prop, vol. 55, no. 11, pp. 3279-3292, 2007.

[4] J. Rius, C. Pichot, L. Jofre, J. Bolomey, N. Joachimowicz, A. Broquetas, and M. Ferrando, "Planar and cylindrical active microwave temperature imaging: numerical simulations," Medical Imaging, IEEE Transactions on, vol. 11, no. 4, pp. 457- 469, 1992.

[5] Guardiola, M.; Capdevila, S.; Blanch, S.; Romeu, J.; Jofre, L., "UWB high-contrast robust tomographic imaging for medical applications," Electromagnetics in Advanced Applications, 2009. ICEAA '09. International Conference on, vol., no., pp.560-563, 14-18 Sept. 2009.

[6] Romeu, J.; Jofre, L., "Truncation Errors in Cylindrical Near to Far Field Transform. A Plane Wave Synthesis Approach," European Microwave Conference, 1992. 22nd, vol.1, no., pp.659-663, Oct. 1992.

[7] (2009) Dielectric Properties of Body Tissues. [Online]. Available: http://niremf.ifac.cnr.it 